\title{
ON GENERALIZATION OF D'AURIZIO-SÁNDOR INEQUALITIES INVOLVING A PARAMETER
}

\section{Li-ChANG Hung AND PEI-Ying Li}

Abstract. In this work, we generalize the D'Aurizio-Sándor inequalities ([2, 4]) using an elementary approach. In particular, our approach provides an alternative proof of the D'Aurizio-Sándor inequalities. Moreover, as an immediate consequence of the generalized D'Aurizio-Sándor inequalities, we establish the D'Aurizio-Sándor-type inequalities for hyperbolic functions.

Mathematics subject classification (2010): 26D15, 26D99.

Keywords and phrases: Inequalities; trigonometric functions; monotonicity.

\section{REFERENCES}

[1] M. Abramowitz and I. A. Stegun, (Eds.), Handbook of Mathematical Functions with Formulas, Graphs, and Mathematical Tables, National Bureau of Standards, Applied Mathematics Series 55, 9th printing, Washington, 1970.

[2] J. D'AuRizIO, Refinements of the Shafer-Fink inequality of arbitrary uniform precision, Math. Inequal. Appl. 17, 4 (2014), 1487-1498.

[3] T. J. Rivlin, Chebyshev Polynomials, Wiley, New York, 1970.

[4] J. SÁNDOR, On D'Aurizio's trigonometric inequality, J. Math. Inequal. 10, 3 (2016), 885-888.

[5] J. SÁNDOR, Extensions of D'Aurizio's trigonometric inequality, Notes on Number Theory and Discrete Mathematics 23, 2 (2017), 81-83. 